Streptococcus agalactiae (GBS). Pur essendo la colonizzazione rettale e/o genitale asintomatica, risulta significativa l'incidenza di endometrite, infiammazione dell'amnios ed infezioni urinarie "peripartum". Altrettanto significativa è l'infezione invasiva del neonato, caratterizzata da sepsi, polmonite, meningite. Questa può realizzarsi nel neonato durante la prima settimana di vita in seguito a trasmissione verticale del microrganismo durante il travaglio e il parto. Oltre a questa infezione "early onset" esiste anche una tardiva "late onset".

La prevenzione primaria dell'infezione da GBS si basa essenzialmente sulla profilassi antibiotica (penicillina) sia nelle donne clinicamente a rischio sia nelle donne con coltura positiva eseguita in $35-37^{\circ}$ settimana di gestazione.

Scopo del nostro lavoro è stata la valutazione in vitro dell'attività del cloruro di benzalconio (CB) nei confronti di GBS, già utilizzato nella terapia locale di vulvovaginiti.

Abbiamo esaminato 52 ceppi di GBS isolati da tampone vaginale di donne gravide. Per valutare l'attività antibatterica di CB, abbiamo misurato per ciascun ceppo MIC e MBC mediante macrodiluizione in brodo secondo NCCLS. Per ciascun ceppo è stata saggiata sensibilità a penicillina, vancomicina, eritromicina, clindamicina mediante sistema Phoenix (Becton-Dickinson).

I valori MIC ottenuti sono compresi tra $6.25-0.39 \mathrm{mg} / \mathrm{ml}$ $\left(\mathrm{MIC}_{90} 3.12 \mathrm{mg} / \mathrm{ml}\right.$ ), quelli MBC tra $12.50-0.78 \mathrm{mg} / \mathrm{ml}$. Per ogni ceppo $\mathrm{MBC}$ è risultata uguale o leggermente più alta rispetto a MIC. Tutti i ceppi sono stati sensibili a penicillina e vancomicina, $10 / 52(19.2 \%)$ resistenti a eritromicina, $12 / 52$ (23\%) a clindamicina e, infine, 40/52 (76.9\%) a tetraciclina. $\mathrm{CB}$ ha mostrato in vitro una buona attività sia batteriostatica che battericida a valori di $3.12 \mathrm{mg} / \mathrm{ml}$. I dati ottenuti hanno evidenziato l'incidenza della resistenza di GBS nei confronti di eritromicina e clindamicina. Il che, a nostro parere, supporta la possibile utilità di una terapia locale con $\mathrm{CB}$ prima del parto ai fini della bonifica delle partorienti.

\title{
ATTIVITÀ ANTIBATTERICA IN VITRO \\ DEL CLORURO DI BENZALCONIO NEI CONFRONTI DI STREPTOCOCCUS AGALACTIAE.
}

Mosca A., lodice MA., Russo F., 'Di Taranto A., ${ }^{2}$ Ciannamea B., Miragliotta G.

Sezione di Microbiologia, Dip. MIDIM,

Università di Bari, P.zza G. Cesare, 70124 Bari;

'Laboratorio di Microbiologia, Ospedale Riuniti di Foggia;

${ }^{2}$ U.O. Microbiologia e Virologia, AUSL LE/I, Lecce

Si calcola che il $10-30 \%$ delle gravide è colonizzato da 